\title{
Proprioception Based Behavioral Advances in a Hexapod Robot*
}

\author{
Haldun Komsuoḡlu $\S$ \\ hkomsuog@umich.edu \\ Ned Moore $\nmid$ \\ ned@cim.mcgill.ca
}

\author{
Dave McMordie $\dagger$ \\ mcmordie@cim.mcgill.ca \\ Martin Buehler $\dagger$ \\ buehler@cim.mcgill.ca
}

\author{
Uluc Saranli $\S$ \\ ulucs@eecs.umich.edu
}

\author{
Daniel E. Koditschek§
}

kod@eecs.umich.edu

\begin{abstract}
§Department of Electrical Engineering and Computer Science, The University of Michigan, Ann Arbor $\dagger$ Center for Intelligent Machines, Ambulatory Robotics Laboratory, McGill University, Montreal, Canada
\end{abstract}

achieves speeds up to $\sim 1.0 \mathrm{~m} / \mathrm{s}$ in these preliminary experiments - resulting from the addition of two sensors and appropriate control modifications.

The original version of this robot (hereafter, RHex 0.0) was sensorless. Its six legs were each driven by an independent hip torque motor that tracked an angular reference trajectory with a PD controller. The six reference trajectories resulted from a single centralized "clock" that generated an alternating tripod gait by splitting out a phase and an "anti-phase" (180 -out) signal copied respectively to the right and left hand tripods. The clock cycle itself was partitioned into "slow" and "fast" components intended to govern a given leg's stance (retraction) and recovery (protraction) modes, respectively. With no available feedback other than the independent local positions and velocities required by each joint's PD controller, RHex 0.0 could truly be said to be performing in a task open loop mode.

The new version, RHex 0.2 , whose performance we describe in this paper, adds to a mechanically improved body, RHex 0.1, a new sensory suite - a body attitude sensor and a ground contact sensor in each leg — along with an approach to modifying the controlling clock signals based on the real time body state information they afford. Beyond documenting the consequently broadened behavioral repertoire, this paper offers a general (and presently somewhat speculative) perspective on how to introduce proprioceptive feedback in clock driven mechanisms founded in part on intuition and in part on insights derived from our previous analyses of Raibert's runners [3,11] and a recently reported stability analysis of a simple (one degree of freedom) clock driven mechanical oscillator [4]. What emerges suggestively in the application of these ideas is the great importance to legged locomotion of managing the phase at which actuator power is expended upon the various constituent oscillatory dynamical systems whose coordination results in net translational progress of the body. Delivering energy to or absorbing energy from the robot's en-

${ }^{*}$ This work is supported by DARPA/SPAWAR Contract N66001$00-\mathrm{C}-8026$. 
vironment at the right phase in the cycle of locomotion events appears to be essential, supporting our conviction that dynamically dextrous robotics amounts to programming work.

A second theme that emerges from this work lies beyond the scope of exposition of the present paper. In the initial communication about RHex [9] we have touched upon the utility for robotics of functional biomimesis - the identification of underlying biological design and control principles that are transferred over to robot hardware and software in the (potentially greatly) altered forms appropriate to the synthetic materials available to engineers. The most salient points of biological inspiration include: (i) compliant legs; (ii) strongly stereotypical "clocked" limb motions; (iii) sprawled posture to enhance stability; and (iv) a stabilizing effect somehow embedded at least in part in the very morphology itself. The force of the first three of these observations is clearly apparent in the form of RHex 0.0, and the validity of the fourth is strongly suggested by the contrast between the robot's significant empirical performance and the utter simplicity and small parameter space of the active controller that elicits that performance. A companion paper [10] surveys aspects of cockroach locomotion that inform these design points and begins to explore the validity of our hypothesized explanations for why they prove to be effective in their impact on the empirical performance of the RHex prototype series. In this paper we, simply presume the validity of these hypotheses and incorporate the new sensory capability in such a way as to enhance their effect. Moreover, we touch upon but do not pursue the connections between the role of proprioception in animals and the inspiration it offers in our work on RHex.

\section{The Physical Robot}

As reported in [9] RHex's design combines the biological inspiration suggested above with the practical engineering principle of robustness through simplicity. The behavioral limitations consequent upon the constrained hardware design of the initial version of RHex are very clear. Since the capabilities of commercially available sensors are improving much more rapidly (thanks largely to breakthroughs in MEMS technology) than are commercially available actuators, we have concentrated in the first year of our work with RHex on incorporating the benefits of more sensors.

Task level control of RHex 0.0 is performed in a feedforward fashion independent of environmental variation. Despite the original algorithm's simplicity, its low computational cost and success over highly uneven terrain, the absence of information about its environment incurs failure quite often. In order to address these problems we have added two specific sensory systems - a leg ground contact sensor; and a body attitude sensor — whose application toward behavioral improvements we will spend the rest of the paper documenting.

\subsection{Previous Versions of RHex}

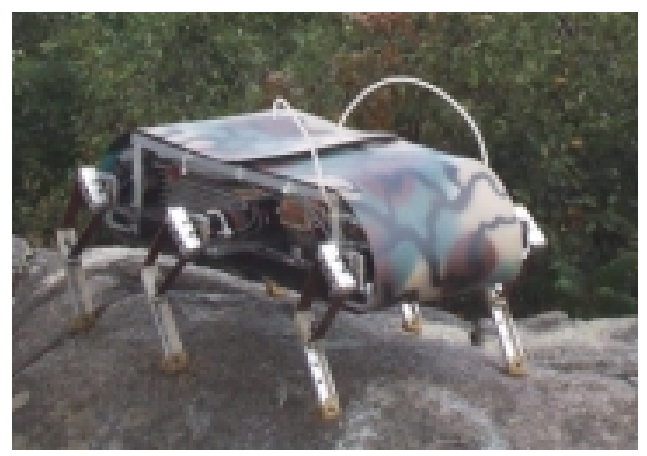

Figure 1: RHex 0.1 in a characteristic outdoor setting.

The first RHex prototype, RHex 0.0 was built in August 1999. Its main body is roughly rectangular in shape and measures approximately $53 \times 20 \times 15 \mathrm{~cm}$. The total weight of the robot is $7.5 \mathrm{~kg}$. The legs, directly coupled to the motors at the hip joints, are made from Delrin rods and are "C" shaped to provide compliance in the radial direction. The reader is directed to [9] for a more detailed account of the physical properties of this machine.

Following this initial prototype, several improvements were made on the robot to improve its mechanical characteristics and behavioral performance. As a result, RHex 0.1 has a slimmer profile with the main body measuring $46.4 \times 21.3 \times 11.4 \mathrm{~cm}$. It weighs $6.8 \mathrm{~kg}$ and has higher capacity batteries for longer autonomous operation. The new leg design, displayed in Figure 1, incorporates a fiberglass four bar linkage providing compliance primarily in the radial direction. A more careful description of these new legs and their effect on RHex's performance is provided in [10].

\subsection{Sensors}

RHex 0.2 uses two new proprioceptive sensors: a body attitude sensor; and a ground contact sensor. In contrast with the primitive sensor suite of the earlier versions of RHex (limited to hip joint shaft angle measurements), these new proprioceptive sensors introduce for the first time the possibility of delivering information about the robot's relationship to its environment.

The body attitude sensor uses the measurement from a 6-dof inertial motion board [6], which yields fore/aft acceleration data, to estimate the angle between the body plane and the gravitational acceleration vector in the sagittal plane. During locomotion, the gravitational forces acting on the accelerometer are coupled with those due to the robot's inertia. The gravitation term is simply the projection of the gravitational acceleration vector onto the body plane, $a_{x}^{G}=g \sin (\alpha)$, where $\alpha$ is the instantaneous body inclination. The motion term, $a_{x}^{A C}$, is a result of robot movements and does not have a DC component during steady state locomotion. Hence, the estimator uses a low-pass Butterworth filter (hereafter, LPF) with cut-off 
frequency at $F_{c}=0.4 H z$ to extract an approximation to the gravity term, $a_{x}^{G} \approx a_{x}^{L P}=L P F\left[a_{x}\right]$, and computes an estimate of the attitude based on the inverse projection formula, $\hat{\alpha}=\arcsin \left(a_{x}^{L P} / g\right)$. Note that for gaits where the body plane is parallel to the surface plane on average, body attitude is equal to the surface inclination.

The second unit, the ground contact sensor, detects individual leg ground contact events. The inertia that hip motors are exposed to is very small during aerial phase of the leg motion relative to that during the ground contact. This leads to comparatively larger PD torque commands during ground contact. This virtual sensor utilizes the estimated motor current as an indirect motor torque measurement to deduce ground contact. Namely, when the $i^{t h}$ motor current, $\hat{I}_{i}$, exceeds a specified threshold, $I_{T D}$, the sensor registers that contact has been made.

\section{Task Level Feedback}

As the task parameters - angle of inclination; height of obstacle; desired speed or endurance - become more extreme, RHex's task open-loop performance degrades, eventually to the point of outright failure.

It seems clear that this degraded performance occurs far earlier than the inevitable limitations of battery energy density and actuator power density should necessitate. How might task level information supplied to the robot in real time permit it to adjust its controls and maintain quality of performance? To answer this question we will first summarize some relevant features of the previously reported open-loop scheme [9], and then propose an account of how proprioceptively driven control parameter adjustment improves performance.

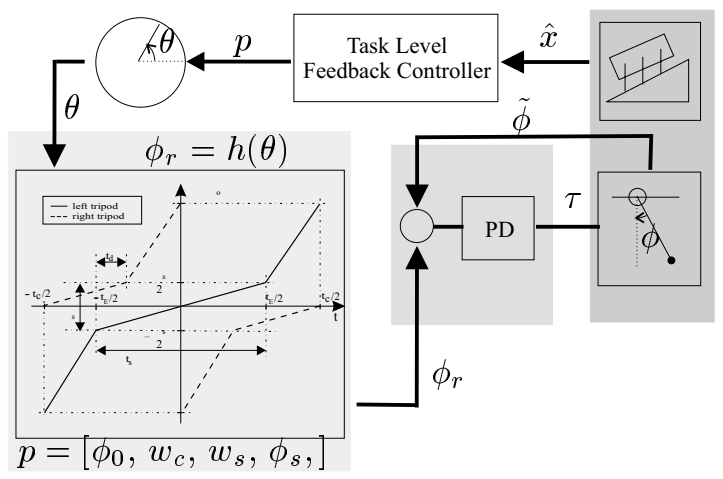

Figure 2: Block diagram of the new RHex controller incorporating proprioception based parameter adjustment. The task level feedback controller adjusts the motion profile parameter, $p$, based on inferences concerning the environment, $\hat{x}$, derived from body measurements. Refer to [9] for the details of the motion profile, $h(\theta)$.

In the companion paper [10] we present evidence acquired both empirically (i.e., by running RHex 0.1 over ground force plates) as well as from extensive simulation that supports the hypothesis that a 2 dof spring loaded inverted pendulum (SLIP) "template" is "anchored" in RHex's 6 dof rigid body dynamics [1] when its control parameters are properly tuned. This hypothesis is depicted in Figure 3. Indeed, it seems to be the case that for slow speeds, the sagittal plane center of mass (COM) trajectory of RHex 0.1 resembles a very stiff SLIP — the rigid inverted pendulum (IP) that biomechanists have proposed as characteristic of walking behavior in most legged animals (Figure 3). In contrast, for fast speeds and appropriate loading conditions, the sagittal plane COM trajectory data of RHex 0.1 [10] exhibits the properties of a "groucho runner" [7] - the motion of a SLIP operating at a periodic orbit tuned in such a fashion as to eliminate any aerial phase. In the sequel, we will simply presume that the mechanics governing RHex's body can indeed be effectively reduced to the SLIP model of Figure 3.

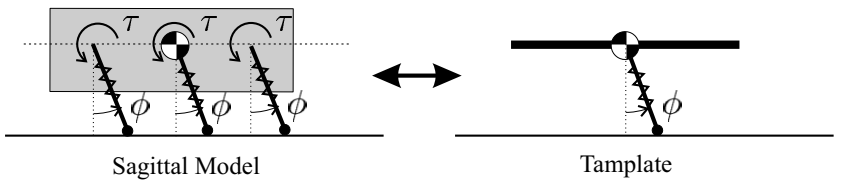

Figure 3: Sagittal plan stance model for a hexapod with one tripod in ground contact (left); a spring loaded inverted pendulum (SLIP) with very large inertial load (right).

The two behaviors targeted in this paper occupy extreme limits in the gait parameter space of the SLIP template. As are all power-autonomous robots, RHex's performance is constrained most severely by the limited power density of its actuators. For inclinations much beyond $10^{\circ}$, the motors typically operate close to or at the speed-torque curve limit during leg protraction (stance) phase unless the clock frequency $1 / T_{c}$ (i.e., the motor shaft reference cycle period depicted in Figure 2), is set very low - typically near $1 \mathrm{~Hz}$. At these stride frequencies, the empirical studies described above [10] suggest that the robot manifests SLIP dynamics characteristic of a very stiff, almost rigid virtual leg. In contrast, on level ground the speed-torque constraints are typically encountered during the no-load retraction phase when the leg must circulate forward through the air to reach its next touchdown before the current stance is complete, limiting the clock frequency below $4 \mathrm{~Hz}$. It follows that on level ground the only way to boost speed is to introduce an aerial phase during which retraction may proceed along the shorter arc below the body, necessitating a fully dynamical SLIP with a very compliant virtual leg. The challenge, then is to develop an approach to proprioceptively driven clock parameter adjustment that can improve performance across the broad spectrum of RHex's operating regime.

The unifying concept across this great breadth of behavior is that an appropriately coordinated periodic trajectory in the robot's joint space can excite an asymptotically sta- 
ble limit cycle of the robot's center of mass. Specifically, we hypothesize that an appropriately tuned "clock" (the periodic hip motor reference trajectory discussed above and depicted in Figure 2) signal yields affordance over the underlying SLIP gait characteristics in a manner that can be systematically manipulated, at least respecting the behavior at (dynamical) steady state. Roughly speaking, the hip actuated SLIP template of Figure 3 exhibits three phase intervals: a support mode where the tripod legs are in ground contact and in slow swing phase; propulsion mode where the legs are in ground contact and in fast swing phase; and finally aerial mode where the tripod legs are in the air.

\section{Empirical Gait Adjustment Studies}

In this section, we propose an intuitive approach to tuning the clock parameters with the goal of adjusting in a task appropriate manner the properties of the resulting template limit cycle. Since there is as yet no available theoretical framework within which to develop these ideas more formally, we are left merely to document their empirical success.

\subsection{Climbing Constant Inclinations}

Pitch destabilization is the main failure mode in climbing constant inclinations: much beyond $10^{\circ}$, the open loop strategy typically pitches the robot backward on its hind legs as depicted in Figure 4a. As discussed above, the slow stride frequencies imposed by actuator power limitations suggest that the SLIP template underlying alternating tripod climbing gaits must operate in a quasi-static mode. These considerations imply that over constant inclines the energy injected by the open-loop controller is both too small in magnitude and channeled into the wrong degrees of freedom - in this specific case into the pitch. Figure 5 depicts the proprioceptively driven adjustment introduced to correct for both of these limitations.

Namely, we adjust the "leg offset" parameter of the open-loop profile, $\phi_{0}$, as a function of surface inclination estimate $\hat{\alpha}$. The inclination compensation rule employed in our experiments is given by $\phi_{0}=-\gamma \hat{\alpha}$ where $\gamma \in \mathbb{R}^{+}$is the compensation parameter. This adjustment has two immediate consequences. First, the amount of energy injected into the system in each step increases, thereby increasing the work that can be done against the vertical (opposing) gravitational potential. Empirical evidence supporting this claim is given in Figure 6. Second, since the support phase extends behind the body as depicted in Figure 4, the average pitch torque generated by the gravitational pull is in the opposite direction to the pitch torque generated by the legs and acts as a pitch stabilizer. Empirical evidence supporting this claim is provided by the greatly decreased

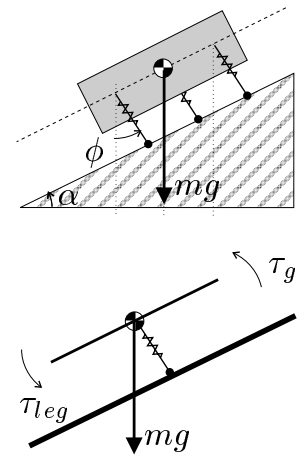

(a)
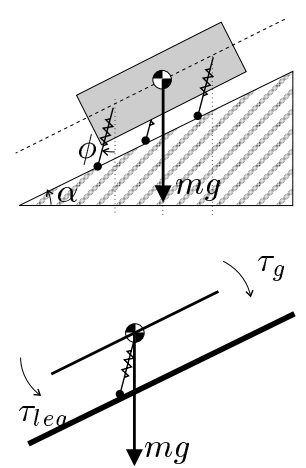

(b)
Figure 4: The quasi-static mechanics of RHex and its presumed (essentially rigid virtual leg) SLIP template when climbing a constant incline. Shifting the touchdown position of the virtual leg allows the gravitationally induced torque, $\tau_{g}$ to counter the pitching disturbance introduced by the motor torque at the hip, $\tau_{\text {leg }}$.
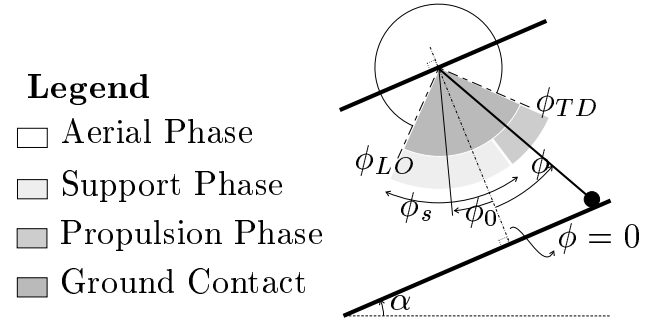

Figure 5: Climbing adjustments to the "clock" parameters, $p$. The "leg offset," $\phi_{0}$ is shifted back as a function of the perceived body inclination.

failure rate (relative to the open loop strategy) as a function of inclination angle.

The optimum compensation parameter is experimentally determined as $\gamma=0.6$. We swept four different inclinations, $\Gamma=\{10.30,16.93,21.71,26.96\}$, at speed setting where the clock period is $T_{c}=0.8 \mathrm{sec}$. For each inclination we ran 10 experiments with the open-loop controller and its compensated version whose performance comparison is summarized in Figure 7.

\subsection{Pronking}

The maximum forward speed reported for the tripod gait is roughly $0.6 \mathrm{~m} / \mathrm{sec}[9]$. As discussed above, our experimental observations suggest that higher forward speeds can be achieved provided an aerial phase is introduced to the robot gait where all 6 legs are in the air for some portion of each strike.

In the open-loop pronking controller all six legs follow a common trajectory command as depicted in Figure 8. The controller switches between two control actions periodically as a function of the clock state, $\theta$, which is defined 


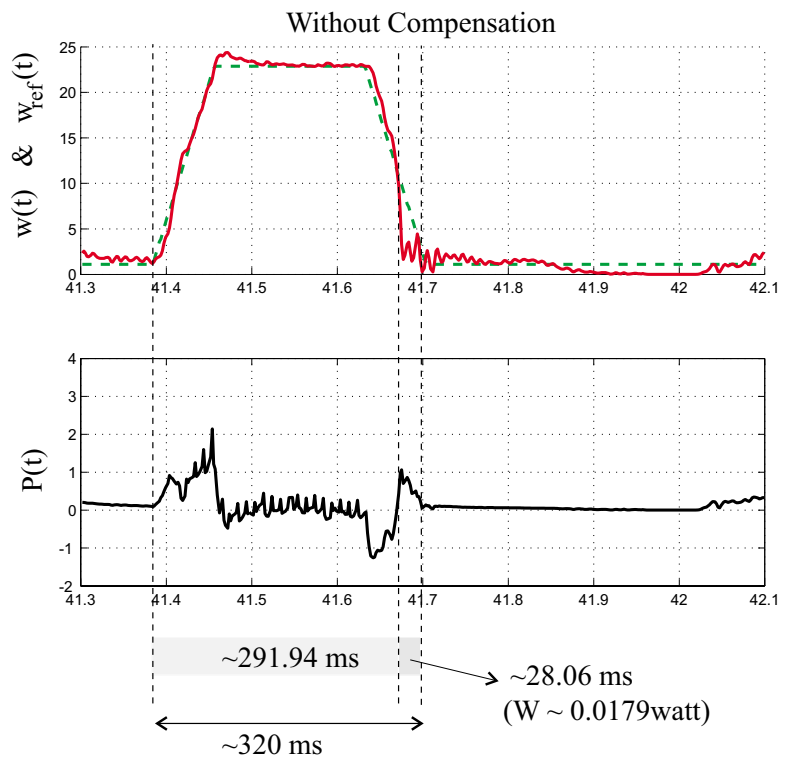

(a)

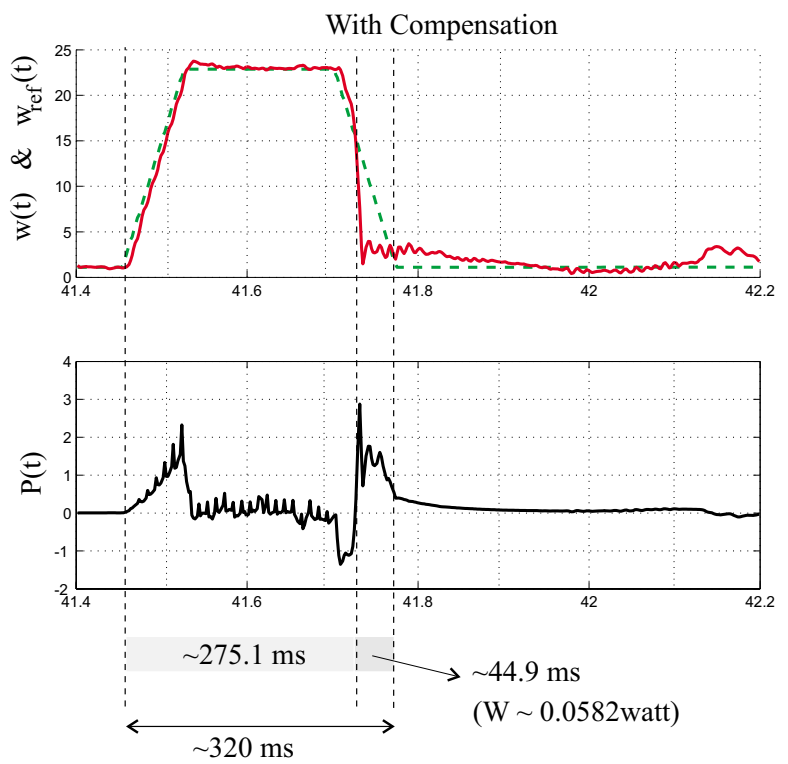

(b)

Figure 6: Hip shaft angle speed reference signal $\left(w_{r e f}\right)$ hip motor position $(w)$ and power $(P)$ data from a hind leg of RHex over a $19.29^{\circ}$ incline: (a) without task level feedback; (b) with task level feedback. Above figures show the reference speed profile (- -) and measured speed (-). The figures below plot the estimated mechanical power output at the hip shaft. Average duration of the support and propulsion phases is indicated for each case. The duration of the propulsion phase is longer for the compensated case yielding $(\sim 33 \%)$ more positive work done in each leg cycle.

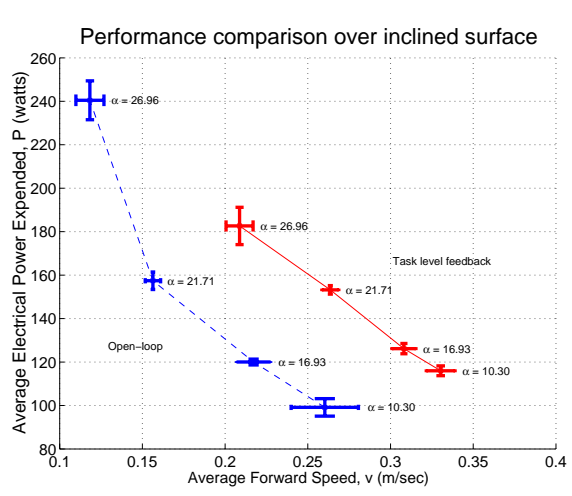

Figure 7: Open-loop controller (dashed blue) and inclination compensated controller (solid red) performance plots. For the two steeper inclinations, the task level feedback controller achieves higher forward speeds and consumes less power than the open loop controller.

by $\dot{\theta}=w_{c}$. In the protraction phase, $\theta \in \Theta_{P}$, the reference motion moves at a constant speed from touchdown angle, $\phi_{T D}$, to lift-off angle, $\phi_{L O}$. The local hip controller's PD gains, $\left(K_{P}^{+}, K_{D}^{+}\right)$are set high in this interval. In the complementary retraction phase, $\theta \in \Theta_{R}:=S^{1}-\Theta_{P}$, the legs are reset back to the touchdown angle with much smaller PD gains, $\left(K_{P}^{-}, K_{D}^{-}\right)$(Figure 8$)$.

In the feedback version of the pronking controller the ground contact sensor synchronizes the controller actions with the motion of the mechanical system. In this imple-

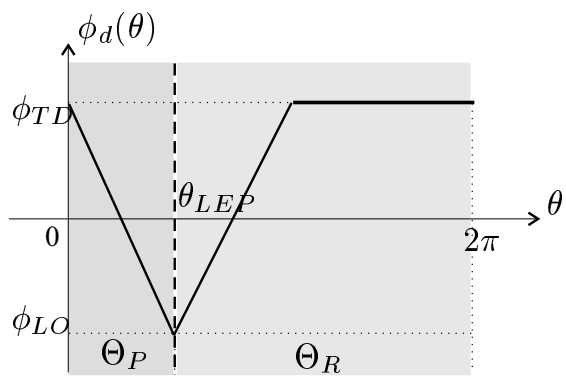

Figure 8: Leg position profile as a function of clock state. In the protraction phase (dark) legs sweep $\left[\phi_{T D}, \phi_{L O}\right]$ at a constant speed. In the retraction phase (light) leg are reset to the touchdown angle, $\phi_{T D}$.

mentation the controller resets the clock state to the beginning of the protraction interval, $\inf \left[\Theta_{P}\right]$, when ground contact is detected. This effectively alters the clock period from stride to stride ensuring the coincidence of stance and protraction phase so that the latter phase can generate propulsion.

The open-loop pronking controller is very inconsistent and introduces severe pitch destabilization. The feedback adjustment just described significantly reduces these destabilizing parasitics. Figure 9 provides a summary of the performance studies at several forward speed settings where the tripod gait and pronking controller with task level feedback are compared. All experiments are run over flat sur- 


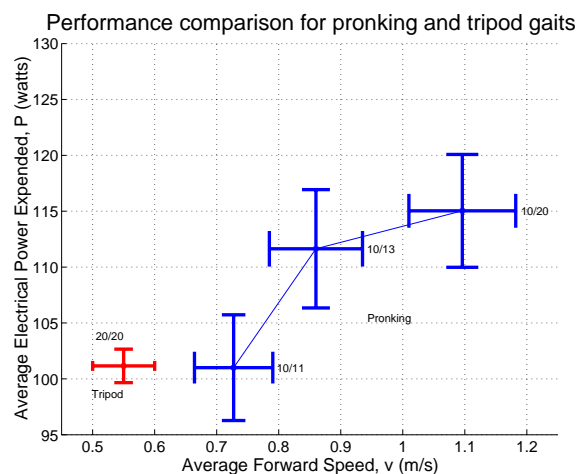

Figure 9: Tripod gait performance (single red) compared to that of the pronking gait (solid blue). Success rates for each experiment set is indicated next to the data boxes. Pronking gait achieves greater forward speeds than the tripod gait.

face, $\alpha=0$. The success rate of the experiment sets are also indicated in the same figure.

\section{Conclusion}

RHex exhibits fast and resilient locomotion behavior over surprisingly challenging terrain. To the best of our knowledge, no legged machine has heretofore been built capable of negotiating at one body length per second ground that is broken and unstable on a scale exceeding the leg ground clearance and approaching the full body dimension itself [9]. These novel capabilities obtain through simplicity of mechanical design and the adoption of open loop control strategies inspired by biomechanists' reports of "collapsed dimension" controllers based on "preflex" stabilized gaits excited by open loop neural pattern generators [1]. However, the pitfalls of "senselessness" are quite obvious (and literal). There are very simple tasks such as climbing simple inclined planes that the fixed open loop controllers cannot achieve. There are higher performance gaits such as confer an aerial phase that almost surely necessitate some degree of task level feedback.

In this paper we document empirically the efficacy of certain proprioceptively tuned open-loop controller adjustments in the context of two very different tasks: quasistatic climbing and dynamical running. Although there is no formal theory yet available to guide the development much less explain the success of these techniques, we are convinced that the underlying unity of concept in our algorithm designs supports the likely utility of the following theoretical framework. High degree of postural freedom can be collapsed onto very low degree of freedom "template" mechanics [1]. Appropriately coordinated rhythmic postural motion can be used to excite mechanically self-stabilizing template limit cycles that achieve the locomotion task [4]. These rhythms and their adjustments must be designed with the goal of synchronizing the rel- ative phase of different sources and absorbers of energy within the closed loop system defined by the body operating into its environment [2]

The foundations of these ideas can be found in Raibert's hoppers wherein the steady state height of a hopping robot was regulated by injecting energy into the system through proper phasing of the controlled actuation [8]. Not surprisingly, analyses of similar but simpler systems have demonstrated the importance of phase synchronization in stabilization [5]. Both the inclination and pronking with stance detection controllers exploit this principle. Notwithstanding the present rudimentary level of formal understanding, it is striking to us how straightforward modifications of our currently primitive controllers can achieve such useful results.

We expect that deeper exploration of biomimetic ideas based upon biomechanical self stabilization and neuromechanical coupled oscillators will yield substantial benefits for robotics.

\section{Acknowledgements}

We thank Byron N. Van Nest for his careful contributions to the development of the inertial motion sensor module.

\section{References}

[1] R. J. Full and D. E. Koditschek. Templates and anchors: Neuromechanical hypotheses of legged locomotion on land. Journal of Experimental Biology, 202(23):3325-3332, 1999.

[2] E. Klavins, H. Komsuoglu, R. J. Full, and D. E. Koditschek. The role of reflexes versus central pattern generators in dynamical legged locomotion. In Neurotechnology for Biomimetic Robots. MIT Press, Boston, 2000.

[3] D. E. Koditschek and M. Bühler. Analysis of a Simplified Hopping Robot. International Journal of Robotics Research, 10(6):587-605, December 1991.

[4] H. Komsuoglu and D. E. Koditschek. Preliminary Analysis of a Biologically Inspired 1-DOF "Clock" Stabilized Hopper. In Proceedings of World Multiconference on Systemics, Cybernetics and Informatics (SCI2000), volume IX, pages 670-675, Orlando, USA, 2000.

[5] H. Komsuoglu and D. E. Koditschek. Proprioception Based Stabilizing Parameter Adjustment Scheme in Clock Stabilized 1-DOF Hopper. Technical report, University of Michigan, Ann Arbor, 2000

[6] H. Komsuoḡlu and B. N. V. Nest. MS-0 hardware reference manual (revision 0). Technical report, University of Michigan, Ann Arbor, 2000 .

[7] T. A. McMahon. Muscles, reflexes, and locomotion. Princeton University Press, Princeton, N.J., 1984.

[8] M. H. Raibert. Legged robots that balance. MIT Press series in artificial intelligence. MIT Press, Cambridge, Mass., 1986.

[9] U. Saranli, M. Buehler, and D. E. Koditschek. Design, Modeling and Preliminary Control of a Compliant Hexapod Robot. In Proceedings of International Conference of Robotics and Automation volume 3, pages 2589-96, San Francisco, CA, USA, April 2000.

[10] U. Saranli, H. Komsuoglu, R. J. Full, M. Buehler, B. Brown, N. Moore, and D. E. Koditchek. Evidence for sprig loaded inverted pendulum running in a hexapod robot. In Seventh International Symposium on Experimental Robotics, 2000.

[11] W. J. Schwind and D. E. Koditschek. Approximating the Stance Map of a 2 DOF Monoped Runner. Journal of Nonlinear Science, 10(5):533-588, 2000 